\title{
PRELIMINARY SEEING MEASUREMENTS FOR SUSI
}

\author{
T.A. TEN BRUMMELAAR, W.J. TANGO, J. DAVIS and R.R. SHOBBROOK \\ Chatterton Astronomy Department \\ University of Sydney, NSW 2006, Australia
}

\begin{abstract}
Some seeing statistics for the Narrabri site of SUSI are presented. These measurements are based on the motion of the wavefront tilt correcting mirrors used in the interferometer. The median seeing for the site to date is 1.3 ".
\end{abstract}

Key words: Tip/Tilt Seeing

\section{Introduction}

The wavefront tilt correction (tip/tilt) servos of the Sydney University Stellar Interferometer (SUSI) have bandwidths of up to $150 \mathrm{~Hz}$ and keep the two beams of the interferometer stable with a standard deviation of 0.1 ". By investigating the mirror positions required to centre the image a direct measurement of the size of the seeing disk is obtained. As well as collecting this seeing data, predictions based on Kolmogorov turbulence for the power spectra and behaviour of wavefront tilt were tested and confirmed.

\section{Measurement Technique}

Two examples of mirror movement are shown in figure (1). The two plots show the mirror movement required to track a stellar image for two separate 2 second samples. The left plot is an example of 'bad' seeing conditions using a sample time of $5 \mathrm{~ms}$ and corresponds to a seeing disc size of $2.0 \pm 0.1$ arcseconds. As a contrast, the plot on the right represents a seeing disc of only $0.9 \pm 0.1$ arcseconds and used a sample time of $1 \mathrm{~ms}$. Note that since the motion of the stellar image is convolved with any mechanical tracking errors the data is to be considered an upper bound on image motion.

These signals can also be used to produce the normalised histograms shown in figure (2). Two examples have been plotted, one for 'bad' and one for 'good'
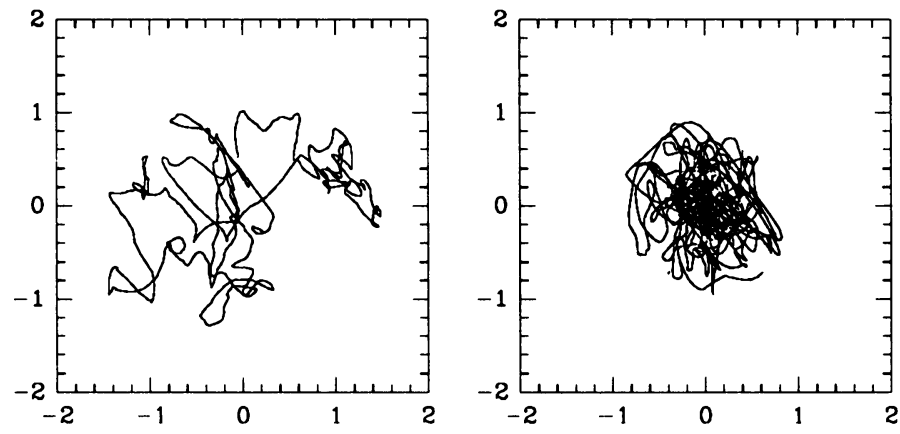

Fig. 1. Stellar image motion in arcseconds. 


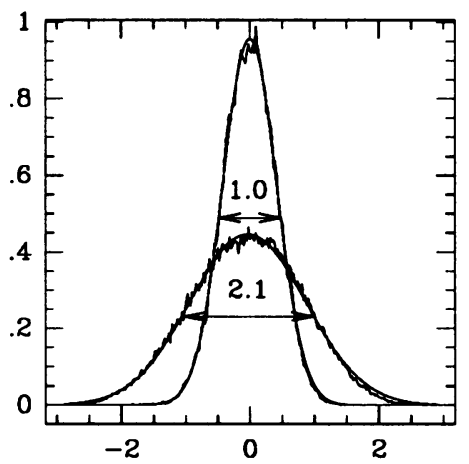

Fig. 2. Seeing disk histograms. Probability Vs Position (arcsec).

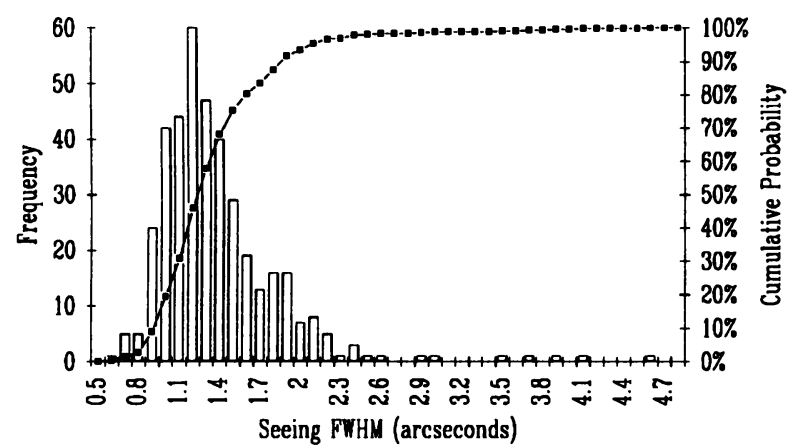

Fig. 3. Seeing Statistics for Narrabri.

seeing conditions. A Gaussian curve fits these plots very well, allowing the measurement of the full width half maximum of the curves, the equivalent of a seeing disc measurement.

\section{Seeing Statistics}

The seeing statistics to date are shown in figure (3). These data were collected over a period of approximately one year. The histogram shows the number of measurements in each bin while the solid line shows a cumulative probability. The mode seeing disk size is 1.2 " while the median value is 1.3 ". The mean is $1.43 \pm 0.02$ ". Seeing of 1.5 " or better has been observed expected $68 \%$ of the time. This compares favourably with other observing sites in Australia.

\section{Wavefront Tilt Power Spectra}

The power spectra of the $a_{2,3}$ Zernike coefficients measured for the star $\alpha$ Car during 'typical' seeing conditions of $1.2 \pm 0.1$ " and an average perpendicular wind speed of 

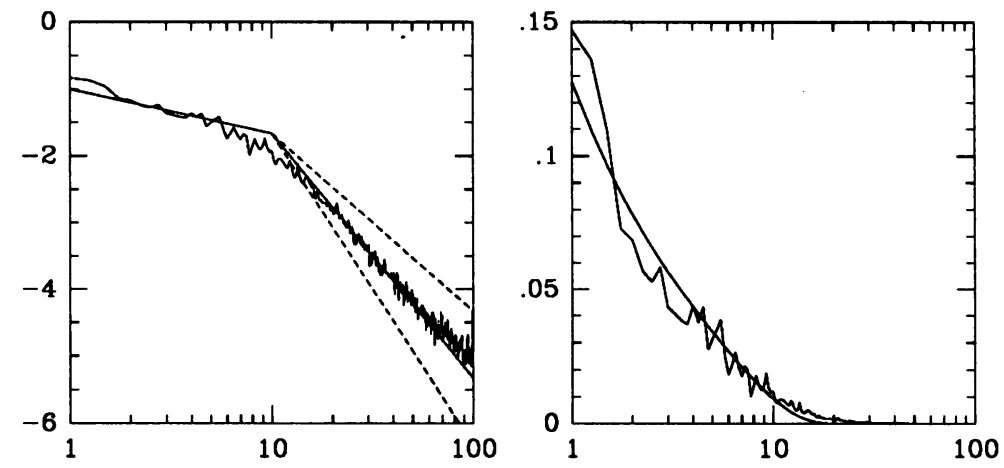

Fig. 4. Wavefront tilt power spectrum. Power Vs Frequency (Hz).

$1.3 \pm 0.2 \mathrm{~m} / \mathrm{s}$ is shown in figure (4). The plot on the left shows the measured spectra along with a simple 'power law' fit. At low frequencies the $-\frac{2}{3}$ power law fits well. This has also been confirmed by Nightingale and Buscher (1991), Doel et al. (1990) and Colavita et al. (1987). At higher frequencies there is some argument about the correct slope; A $-\frac{8}{3}$ (Hogge and Butts, 1976), a $-\frac{11}{3}$ (Tango and Twiss (1980), Fields (1983) and Acton et al. (1992)) and a $-\frac{14}{3}$ (ten Brummelaar, 1992) slope are all displayed. Clearly the $-\frac{11}{3}$ slope fits best. The other plot shows the same data fitted to a more exact formulation (ten Brummelaar, 1992) displayed on a linear scale. While the high frequency part has not been fitted well, the plot on a linear scale shows that, in the context of the tilt correction servo, it contains so little power that it may be ignored. Work continues in this area.

\section{Acknowledgements}

One of us (TtB) acknowledges the support of an Australian Postgraduate Research Award. The SUSI project is funded by the Australian Research Council and the University of Sydney. The support of the Pollock Memorial Fund and the Science Foundation for Physics within the University of Sydney is also gratefully acknowledged.

\section{References}

Acton, D.S., Sharbaugh, R.J., Roehrig, J.R. and Tiszauer, D.: 1992, Applied Optics 31, 4280-4284 ten Brummelaar, T.A.: 1992, Taking the Twinkle Out of the Stars: An Adaptive Wavefront Tilt Correction Servo and Preliminary seeing Study for SUSI, Chatterton Astronomy Department, University of Sydney, Ph.D. Thesis

Colavita, M., Shao, M. and Staelin, D.H.: 1987, Applied Optics 26, 4106-4112

Doel, A.P., Dunlop, C.N., Major, J.V., Myers, R.M., Purvis, A. and Thompson, M.G.: 1990, S.P.I.E. Advanced Optical Telescopes IV 1236, 179-192

Fields, D.R.: 1983, Applied Optics 22, 645-647

Hogge, C.B. and Butts, R.R.: 1976, IEEE Trans. Antennas Propagat. AP-24, 144-154

Nightingale, N.S. and Buscher, D.F.: 1991, Mon. Not. R. astr. Soc. 251, 155-166

Tango, W.J. and Twiss, R.Q.: 1980, Progress in Optics XVII, 239-277 